\title{
ADRENALINE SENSITIVITY OF PERIPHERAL BLOOD VESSELS IN HUMAN HYPERTENSION
}

\author{
BY \\ ROBERT S. DUFF \\ From the Cardiological Department, St. Bartholomew's Hospital \\ Received January 25, 1956
}

The exact cause of the increased peripheral resistance in chronic arterial hypertension is unknown. It is not due to generalized overactivity of the vasomotor nerves for Prinzmetal and Wilson (1936), Pickering (1936a), and Stead and Kunkel (1940) showed that the vasoconstriction responsible for essential hypertension is not neurogenic. Search for abnormal pressor agents in the blood stream has generally been unsuccessful (Hülse, 1924: Aitken and Wilson, 1935; Pickering, $1936 b$ ). Likewise, with rare exceptions such as phæochromocytoma, no increase in the level of naturally occurring pressor hormones has been found (Engel and von Euler, 1950; Raab and Gigee, 1954). Organic narrowing of the arterioles cannot be responsible, for this occurs only in the later stages and in restricted portions of the vasculature (Moritz and Oldt, 1935).

There remains, however, one further possibility of accounting for the generalized reduction of peripheral vascular calibre in hypertension: increased sensitivity of the terminal arterioles to normal constrictor stimuli. The present paper describes a method of selectively measuring the sensitivity of blood vessels and submits observations on groups of subjects with normal and raised blood pressures, respectively.

\section{SUBJECTS}

Findings in 39 subjects with normal blood pressures (under 150/90 mm. Hg) have been contrasted with those of 25 hypertensive patients. The normals were mostly healthy medical student volunteers but included some patients with non-hypertensive disorders of the circulation, such as Raynaud's disease. Their ages ranged from 20 to 51 years.

Only patients with persistent elevation of blood pressure over $200 / 100 \mathrm{~mm}$. $\mathrm{Hg}$ were included in the hypertensive group. After clinical, electrocardiographic and radiological assessment each case was graded according to the condition of the heart, retinæ, coronaries, and kidneys:

(a) benign-when these structures were affected but little or not at all;

(b) progressive-when one or more tissue had severe or perceptibly increasing damage; or

(c) malignant-when papilloedema associated with gross vascular lesions were present.

Important characteristics of the various groups of subjects are summarized in Table I.

TABLE I

SUBJECTS

\begin{tabular}{c|c|c|c|c}
\hline \multicolumn{1}{c|}{ Group } & Men & Women & All & Mean age \\
\hline With normal pressure & 30 & 9 & 39 & 29 \\
With raised pressure & 7 & 18 & 25 & 50 \\
\hline
\end{tabular}




\section{MeTHODS}

Throughout each test the subject reclined comfortably on a couch in a room maintained at a temperature of $23 \pm 1^{\circ} \mathrm{C}$. At regular half-minute intervals the blood flow in both hands was measured separately by venous occlusion plethysmography, following a standard procedure (Duff, 1952).

Under local anæsthesia a continuous infusion into the brachial artery on one side was set up, using a power-driven double-channel apparatus delivering saline at a constant rate. At regular 4 -minute intervals plain saline $(0.9 \%(\mathrm{w} / \mathrm{v}) \mathrm{NaCl}$ solution) was alternated with solutions of adrenaline sufficiently diluted to ensure minimal responses confined to the infused hand. Each solution was freshly prepared by adding synthetic L-adrenaline tartrate (B.D.H.) to saline containing a trace of ascorbic acid to prevent catechol deterioration. Every subject was tested with two or more of the following adrenaline concentrations: $1 / 64,1 / 16$ and $1 / 8 \mu \mathrm{g} . / \mathrm{min}$. The highest dose used was therefore less than 5 per cent of that required systemically to cause either subjective symptoms, or perceptible effects on heart or blood pressure. Under these conditions subjects generally were comfortable throughout the period of observation, and remained unaware of the infusions.

Independent measurements of the blood flow in the test hand and in the opposite control hand are obtained while a continuous infusion is given into the brachial artery on the test (usually the right) side. When saline alone is being infused the variation in blood flow on one side during a given period is approximately the same as that on the other side, but when an effective amount of adrenaline is added, this close parallelism is disturbed by the resulting vasoconstriction in the test hand. If $A$ and $B$ are the mean blood flows in the test hand during successive periods of time and $a$ and $b$ are corresponding values for the other hand, then $A / B$ approximately equals $a / b$. When three of these values are known the fourth can be calculated: e.g. $B=A b / a$. If saline alone is infused during the first period (corresponding blood flows in the two hands averaging $A$ and $a$ ) and adrenaline is added during the second period (corresponding blood flows being $B$ and $b$ ), then an estimate $E$ of what the blood flow in the test hand would have been had the adrenaline been ineffective is obtained by $E=A b / a$. Therefore the net effect of the adrenaline is the difference between the observed mean $B$ and the expected mean $E$, after due allowance for experimental error. When the observed mean $B$ is less than the calculated mean $E$ (derived from three observed control means) it may be inferred that the adrenaline has caused vasoconstriction in the test hand. A useful measure of this adrenaline effect is given by $B-E / E \times 100$. It has been shown (Duff, 1952) that the amount of adrenaline required to reduce this expression by 25 per cent may be taken as the threshold of the hand vessels to adrenaline.

\section{RESULTS}

Subjects with Normal Blood Pressure. Because of the wide range of response of healthy subjects a large number with normal pressure had to be studied. While the majority exhibited significant vasoconstriction only when the adrenaline levels exceeded $1 / 16 \mu \mathrm{g} . / \mathrm{min}$., a few reacted with $1 / 16$ or less. A common type of response is portrayed in Fig. 1. The synchronous fluctuation of the blood flow in the two hands (due to bilateral influences unrelated to the infusion) is seen to persist even when adrenaline is being infused into one hand. With the smallest dose of adrenaline $(1 / 64 \mu \mathrm{g} . / \mathrm{min}$.) the greatest individual constriction was 17 per cent and the mean response of the group was under 5 per cent (Table II). With $1 / 16$. adrenaline only 4 of the 20 subjects had significant vasoconstriction, the mean for the group being 11 per cent. With the highest adrenaline level $(1 / 8 \mu \mathrm{g} . / \mathrm{min}$.) 13 of the 23 normal subjects had over 25 per cent vasoconstriction, while 10 had under 25 per cent; the mean for the group was 25 per cent constriction (Table II). Statistical parameters of these observations are given in this Table.

Hypertensive Subjects. When subjects with raised blood pressures were compared under identical conditions with healthy subjects it became apparent that the same stimuli (adrenaline infusions) tended to constrict the hypertensives more. The responses of a woman with a blood 


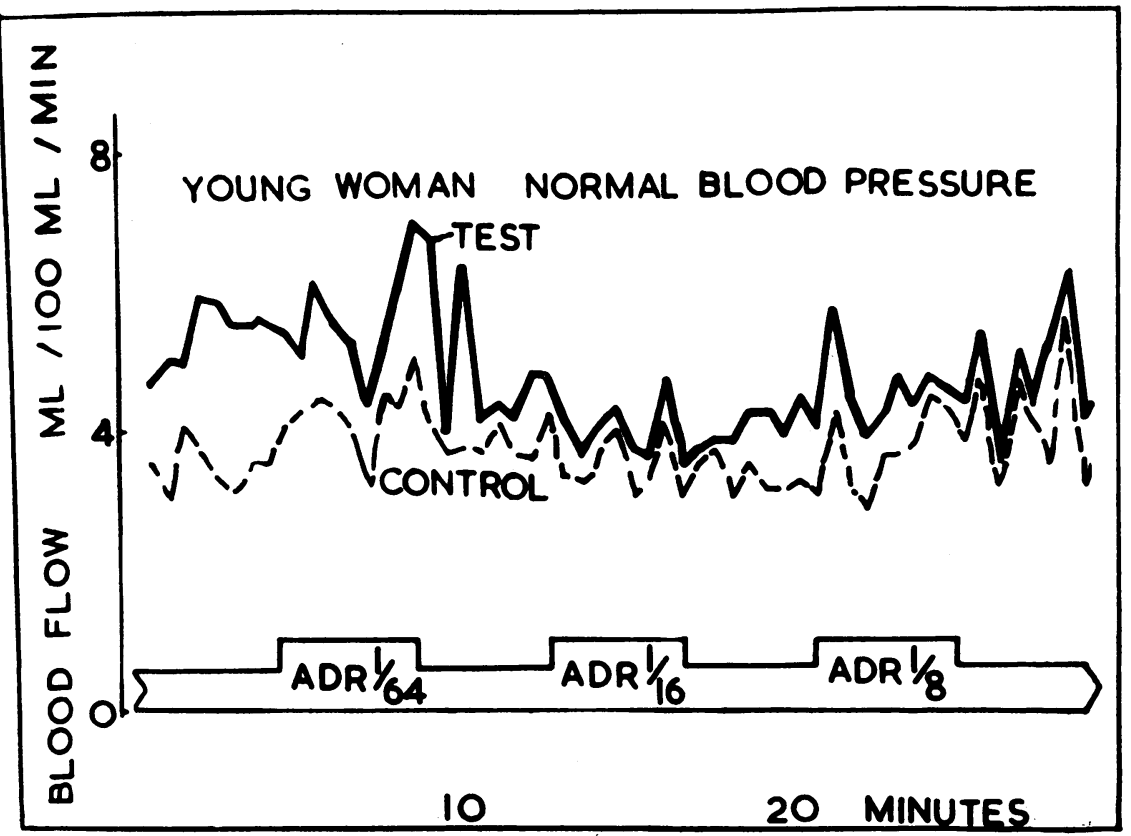

FIG. 1.-Blood flow in the infused test hand (heavy continuous line) and in the control hand (interrupted faint line) of a healthy subject during intrabrachial infusions of adrenaline, $1 / 64 \mu \mathrm{g} ., 1 / 16 \mu \mathrm{g}$., and $1 / 8 \mu \mathrm{g} . / \mathrm{min}$. None of these infusions caused independent vasoconstriction in the test hand.

TABLE II

Mean Adrenaline Vasoconstriction of Groups of Subjects with Normal or with Raised Blood Pressure

\begin{tabular}{|c|c|c|c|}
\hline $\begin{array}{l}\text { Adrenaline } \\
\text { level } \\
(\mu g . / m i n .)\end{array}$ & & $\begin{array}{l}\text { With normal } \\
\text { pressure }\end{array}$ & $\begin{array}{c}\text { With raised } \\
\text { pressure }\end{array}$ \\
\hline $1 / 64$ & $\begin{array}{l}\text { Number of subjects } \\
\text { Mean constriction } \\
\text { Standard error }\end{array}$ & $\begin{array}{r}10 \cdot 0 \\
4 \cdot 7 \\
2 \cdot 4\end{array}$ & $\begin{array}{r}10 \cdot 0 \\
13 \cdot 8 \\
6 \cdot 7\end{array}$ \\
\hline $1 / 16$ & $\begin{array}{l}\text { Number of subjects } \\
\text { Mean constriction } \\
\text { Standard error }\end{array}$ & $\begin{array}{r}20 \cdot 0 \\
11 \cdot 3 \\
3 \cdot 8\end{array}$ & $\begin{array}{r}20 \cdot 0 \\
33 \cdot 6 \\
5 \cdot 0\end{array}$ \\
\hline $1 / 8$ & $\begin{array}{l}\text { Number of subjects } \\
\text { Mean constriction } \\
\text { Standard error }\end{array}$ & $\begin{array}{r}23 \cdot 0 \\
25 \cdot 0 \\
5 \cdot 6\end{array}$ & $\begin{array}{r}23 \cdot 0 \\
52 \cdot 8 \\
4 \cdot 4\end{array}$ \\
\hline
\end{tabular}

pressure of 260/130 are shown in Fig. 2. Clearly all these adrenaline infusions caused independent constriction of the test hand. As in the case of the healthy subjects the range of response of those with hypertension was fairly wide, some of the latter having little or no constriction even with the highest dose of adrenaline. However, there was a particular tendency for those with more severe grades of hypertension to exhibit increased sensitivity to adrenaline. The responses of a woman with malignant hypertension are illustrated in Fig. 3. Even the lowest adrenaline concentration caused 40 per cent vasoconstriction in the test hand. The individual responses at each dose level are summarized in Table II. With $1 / 64 \mu \mathrm{g}$. adrenaline the mean response of 10 hypertensive subjects was 


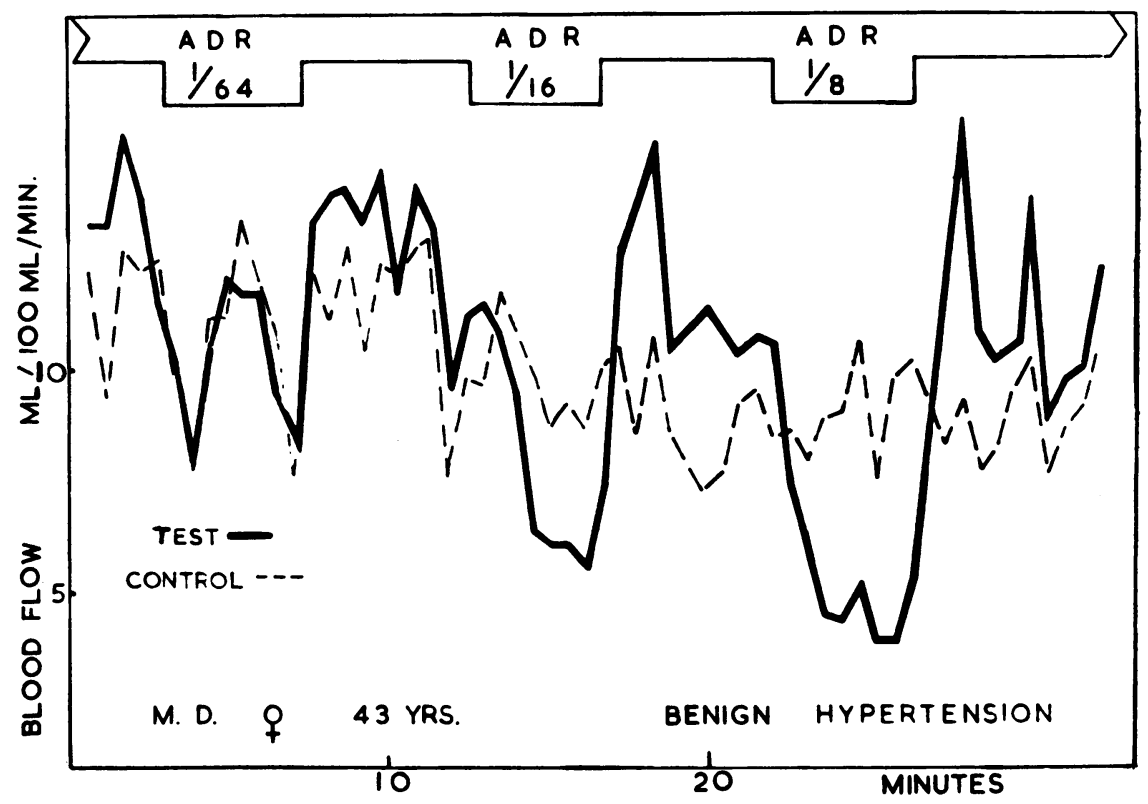

FIG. 2.-Effects of adrenaline infusions on blood flow in the test hand of a subject with hypertension. Significant vasoconstriction resulted from two of the infusions.

13.8 per cent, almost three times that of the normal group. With $1 / 16 \mu \mathrm{g}$. adrenaline 14 of the 20 hypertensive cases had significant constriction, compared with 4 of the normals. As a group the mean response of those with hypertension was about thrice that of the normals. With the highest adrenaline level ( $1 / 8 \mu \mathrm{g}$.) only 3 of the 23 patients failed to exhibit appreciable vasoconstriction, in contrast with the failure of 10 of the healthy subjects to respond.

The data are summarized in Table II; the differences between the responses of the normal and hypertensive subjects are statistically significant at the $1 / 16$ and $1 / 8 \mu \mathrm{g}$. levels. The mean responses of the two groups of subjects with each adrenaline level are contrasted in Fig. 4.

An expression for the mean vascular sensitivity of a given individual may be obtained by adding the separate responses at each adrenaline level. This is conveniently done by allotting to the various responses a value inversely proportional to the strength of the stimulus as in the following schema.

\begin{tabular}{ccccc} 
& \multicolumn{2}{c}{ Adrenaline concentration } & Unit of \\
& $1 / 64$ & $1 / 16$ & $1 / 8 \mu \mathrm{g} . / \mathrm{min}$. & sensitivity \\
Percentage vasoconstriction & & & under 21 & 0 \\
$(B-E / E \times 100)$ & & & $21-40$ & 1 \\
& & $21-40$ & $41-60$ & 2 \\
& $21-40$ & $41-60$ & $61-80$ & 3 \\
& $41-60$ & $61-80$ & $81+$ & 4
\end{tabular}

This scale lays proper emphasis on the threshold response to minimal stimulation. The mean vascular sensitivity of each tested subject was calculated in accordance with the above scale. Those with normal blood pressure ranged from 0 to $3 \cdot 0$, with a mean of 0.7 for the 39 subjects. Those with hypertension ranged from 0 to $3 \cdot 5$, with a mean of $2 \cdot 0$. Thus the mean sensitivity of the hypertensive group was almost three times the normal. The degree of sensitivity was seen (Fig. 5) to be generally related to the severity of the hypertensive process. Some cases of benign hypertension fell within the normal range, but most of those graded progressive were hypersensitive, and the greatest sensitivity was found in those with malignant hypertension.

These experiments have clearly demonstrated a significant difference in vascular sensitivity between two groups of subjects, with normal and raised blood pressures, respectively. Before 


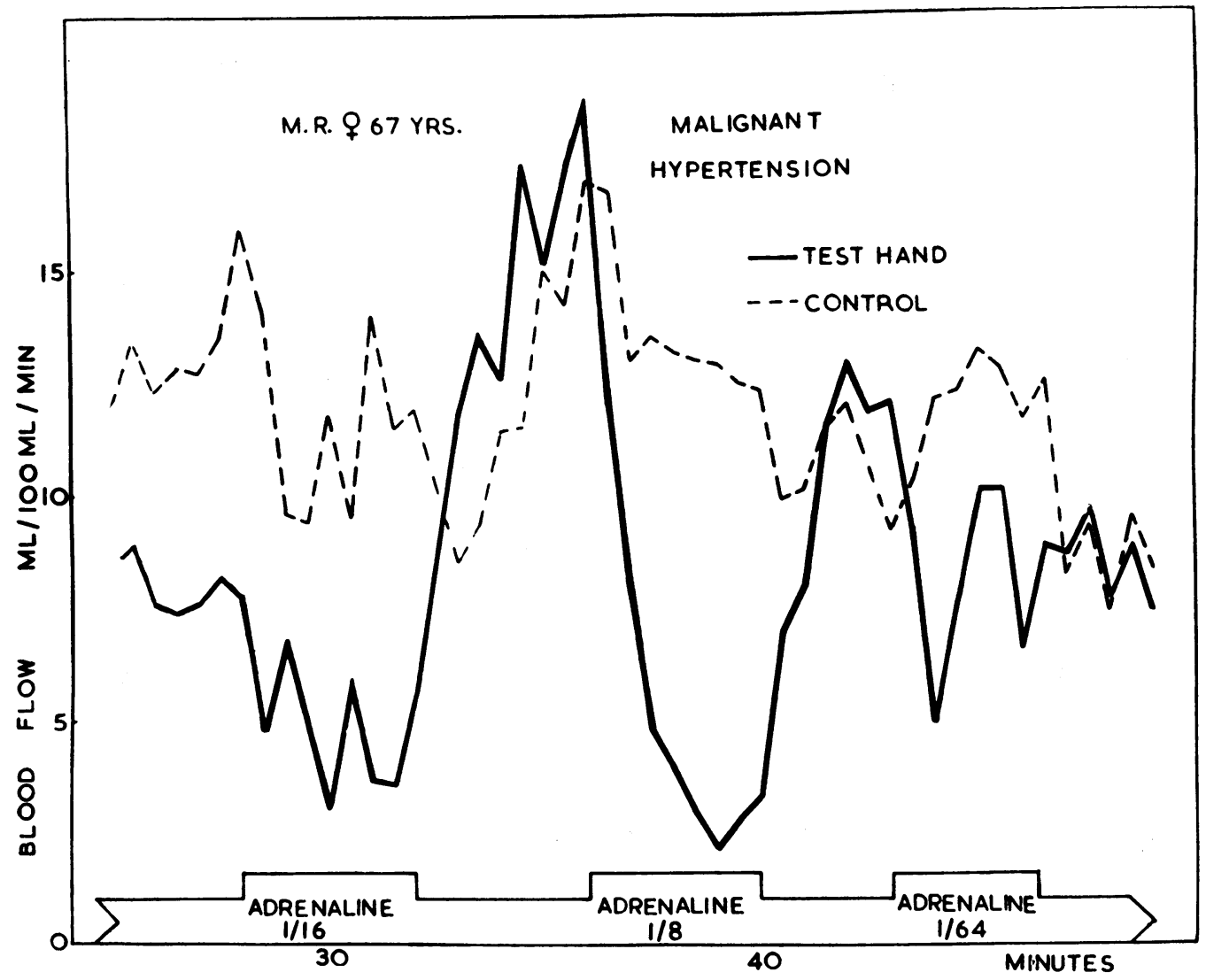

FIG. 3.-Vasoconstriction with adrenaline, even in the low concentration of $1 / 64 \mu \mathrm{g} . / \mathrm{min}$. in a subject with malignant hypertension.

concluding that the increased sensitivity of the latter is directly related to the hypertension it is necessary to consider critically if the observed difference in sensitivity between the two groups can be attributed to anything other than the hypertension. In addition to blood pressure, the two populations differed in three main respects: age, sex, and profession.

Many of the healthy subjects were younger than those with hypertension, the mean ages of the groups being 29 and 50 years, respectively (Table I). However, about one in four of the former was over 35 and one in three of the latter under 45 years of age. Moreover, the individual responses seemed to be quite unaffected by the age of the subjects, some of the most sensitive being younger than others with less vascular sensitivity. Indeed, the proposition that age has a bearing on vascular sensitivity is clearly not favoured by the haphazard relationship of the two factors.

Most of the healthy subjects were men while most of those with hypertension were women. This might therefore suggest that vascular sensitivity may be greater in women. Examination of individual responses, however, revealed that the vascular sensitivity of eight of the nine normal women was below the normal mean, all but one of those exceeding the mean being men. Moreover, in the hypertensive group four of the seven men, including one with malignant hypertension, had above average sensitivity. There is therefore no support for the hypothesis that sex influences vascular sensitivity.

The remaining factor-occupation-clearly differentiates the two groups, most of the normals being medical students. It could hardly be maintained, however, that medical students are unrepresentative of the general population with respect to such a characteristic as vascular sensitivity to adrenaline. 


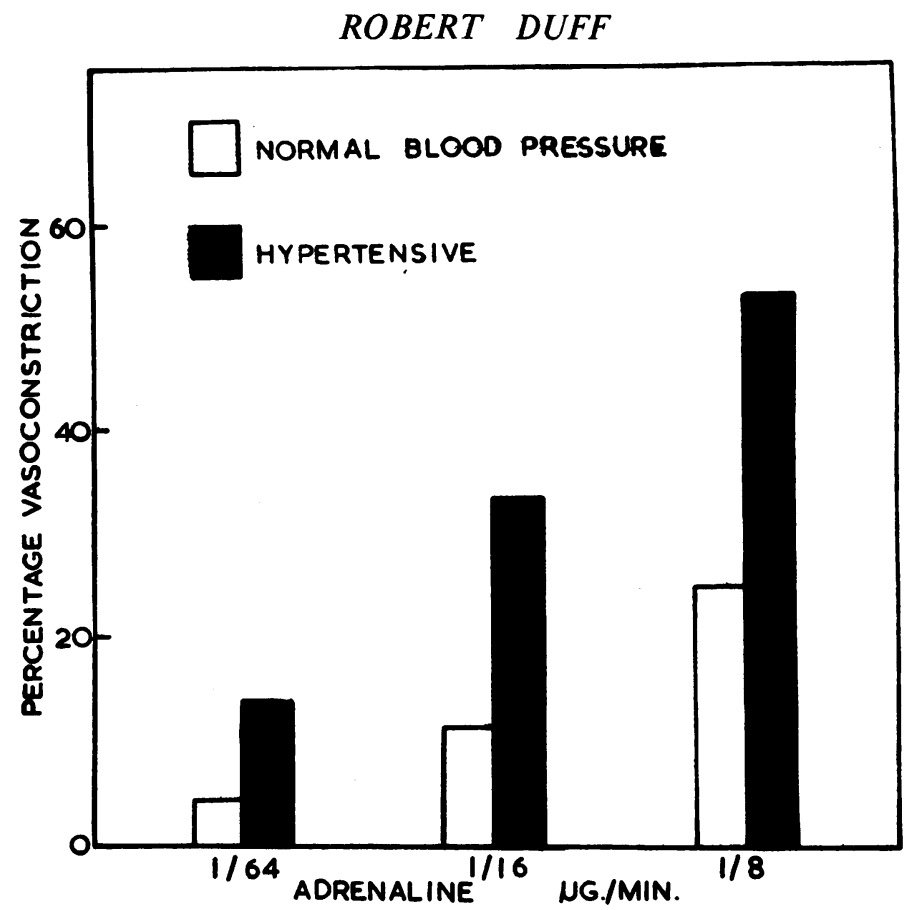

Fig. 4.-Comparison of the constrictor effects of three concentrations of adrenaline in two groups of subjects, with normal and raised blood pressures, showing the greatly increased responses of the latter.

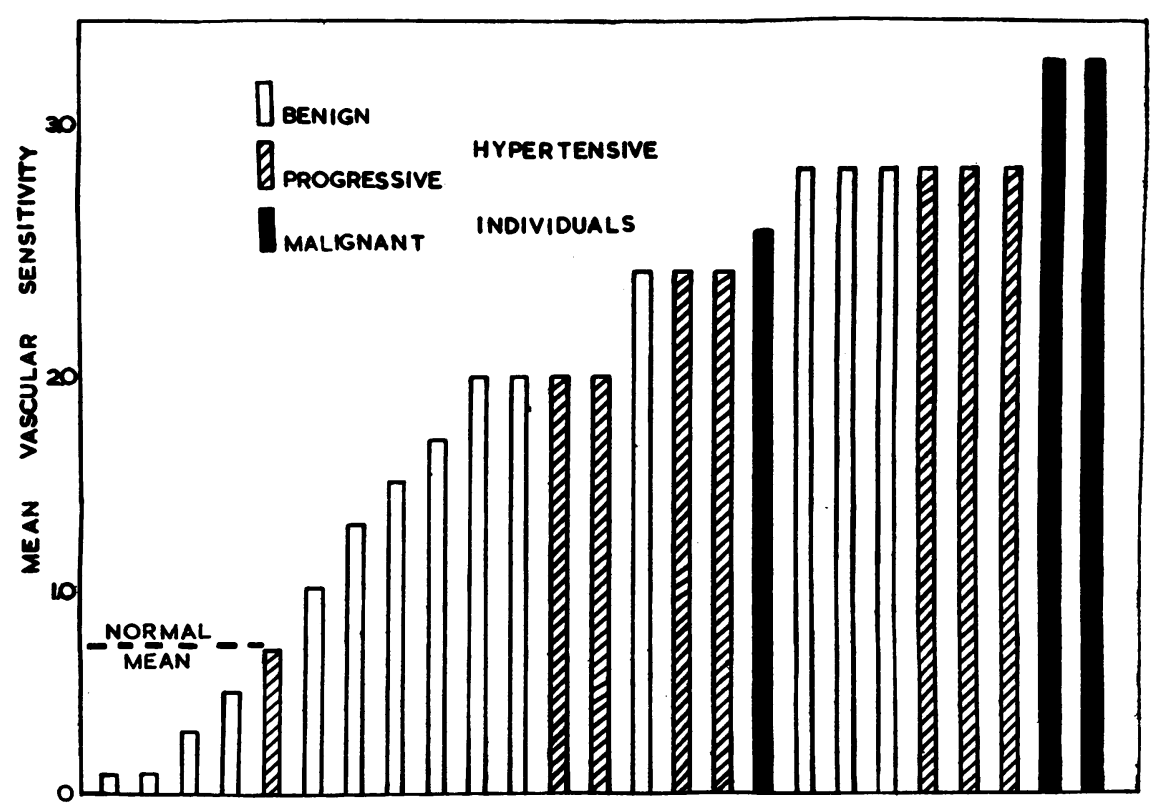

FIG. 5.-Mean vascular sensitivity of subjects with hypertension. All but 5 exceed the normal mean, and in general, those with progressive or malignant hypertension are particularly sensitive to adrenaline. 
These considerations do not exclude the possibility that the observed differences in vascular response between the two groups of subjects have a fortuitous relationship to the occurrence of hypertension, though they make it extremely unlikely. The inference that increased vascular sensitivity has something to do with pathological elevation in blood pressure is probably justified.

\section{Discussion}

A tendency for adrenaline to cause increased peripheral constriction in hypertension has been demonstrated by these experiments. This is exemplified both by the higher incidence of significant vasoconstriction with small doses of adrenaline in those with hypertension and by their greater response as a group at all adrenaline levels (Table II). Direct evidence has therefore been provided of a general relationship between vascular sensitivity and human hypertension. Reports hitherto published are lacking in comparable quantitative data, but indirect evidence of increased sensitivity to adrenaline in essential hypertension has been described in the nailfold capillaries (Greisman, 1952) and in the bulbar conjunctiva (Lee and Holtze, 1951).

Detailed consideration of individual rather than group responses revealed two important findings. A few of the healthy subjects gave responses far greater than the group as a whole. This may merely express the inherent variability of biological material, but it is equally possible that these more sensitive subjects are indeed pre-hypertensive. Neither speculation conflicts with the concept that hypertension is a "graded characteristic" in the general population (Hamilton et al.,1954). It is also noteworthy that a few subjects with benign hypertension had adrenaline responses within the normal range, although most of those with progressive and all with malignant hypertension had increased sensitivity. It may therefore be necessary to recognize two distinct categories of hypertension: (1) clinically mild, without demonstrable abnormality of vascular function; and (2) clinically severe or progressive, with vascular hypersensitivity. It is a common observation that some cases of marked and persistent hypertension may be attended by comparatively little reduction of health and longevity, in striking contrast to the rapid deterioration of others with equal or even lower levels of blood pressure. Until patients have been observed over longer periods it is open to speculation whether the onset of deterioration in hitherto benign cases is related to changes in vascular sensitivity.

The failure to find excessive amounts of adrenaline in essential hypertension has already been noted. The present study provides a basis for the generalized constriction of peripheral arterioles in hypertension in the presence of normal amounts of adrenaline (or other pressor hormones).

Hypersensitivity was not demonstrated in a group of hypertensive cases, all of which were " benign ". In the remainder the abnormality of vascular function tended to be proportional to the severity of the disease when graded according to structural damage. This may suggest that hypersensitivity is the result rather than the cause of prolonged and excessive rise in blood pressure. Increased sensitivity to adrenaline might be due to compensatory hypertrophy of the muscular coat of the terminal arterioles. Alternatively the abnormality of vascular function might be related to those more severe arteriolar lesions responsible for clinically perceptible damage in the retinæ, heart, or kidneys.

The technique of the present study selectively measures the behaviour of the terminal arterioles themselves. It must therefore be emphasized that the findings, being much more specific, bear little relation to tests of generalized cardiovascular function, such as that of Hines and Brown (1936).

The question of vascular sensitivity is of much more than academic interest. The demonstration of a functional abnormality of the vessels themselves underlines the purely palliative character of therapy based on chemical or surgical interference with the autonomic nervous system. If subsequent investigation elucidates the nature of the vascular abnormality and its relation to the production and progress of clinical hypertension, this may eventually provide a rational basis for the prevention and treatment of the disease. 


\section{SUMMARY}

A method of measuring in man the sensitivity of selected peripheral blood vessels has been described. This is based on the quantitative measurement, by venous occlusion plethysmography, of the change in blood flow resulting from the introduction of minimal chemical stimuli into the brachial artery.

In 39 subjects with normal, and 25 with pathologically raised blood pressures, the responses of blood vessels in the hands to adrenaline infusions of $1 / 64,1 / 16$, and $1 / 8 \mu \mathrm{g}$. $/ \mathrm{min}$. have been determined under controlled conditions.

As a group the vascular sensitivity of the hypertensive group has been shown to be almost three times that of the normal group.

In a proportion of subjects with hypertension the vascular sensitivity was found to be normal. In the remainder it was shown to be related to the severity of the hypertensive process.

On the basis of these findings, the recognition of two distinct categories of hypertension has been envisaged; one benign, with normal vascular function, and the other progressive, with vascular hypersensitivity.

I am most grateful to Dr. Geoffrey Bourne and Dr. Graham Hayward both for making patients available and for valuable advice and encouragement; and to Professor Sir James Paterson Ross and Professor Henry Barcroft for providing apparatus and laboratory facilities at St. Bartholomew's and St. Thomas's Hospitals. Some of the data on healthy subjects was obtained in collaboration with Dr. Jean Ginsburg to whom I am indebted for valuable help and criticism. The generous cooperation of patients at St. Bartholomew's Hospital and of medical students at St. Thomas's Hospital made this study possible.

\section{REFERENCES}

Aitken, R. S., and Wilson, C. (1935). Quart. J. Med., 4, 179.

Duff, R. S. (1952). J. Physiol., 117, 415.

Engel, A., and von Euler, U. S. (1950). Lancet, 259, 387.

Greisman, S. E. (1952). J. Clin. Invest., 31, 782.

Hamilton, M., Pickering, G. W., Roberts, J. A. F., and Sowry, G. S. C. (1954). Clin. Sci., 13, 11.

Hines, E. A., jun., and Brown, G. E. (1936). Amer. Heart J., 11, 1.

Hülse, G. (1924). Z. Ges. exp. Med., 39, 413.

Lee, R. E., and Holtze, E. A. (1951). J.' Clin. Invest., 30, 539.

Moritz, A. R., and Oldt, M. R. (1935). Amer. J. Path., 11, 885.

Pickering, G. W. (1936a). Heart, 2, 209.

- (1936b). Clin. Sci., 2, 185.

Prinzmetal, M., and Wilson, C. (1936). J. Clin. Invest., 15, 63.

Raab, W., and Gigee, W. (1954). Circulation, 9, 592.

Stead, E. A., and Kunkel, P. (1940). J. Clin. Invest., 19, 25. 